\title{
Improving access to psychological therapies: implications for mental health care in general practice
}

A total of 3600 new accredited psychological therapists are being trained and employed in primary care over the 2008-2011 period as part of the Improving Access to Psychological Therapy (IAPT) programme. ${ }^{1}$ This is the largest investment ( 170 million) in primary care mental health ever, and comes with a number of preconditions: practitioners will be employed in teams; have routine supervision; collect patient-rated outcome measures at each contact (including the Patient Health Questionnaire-92 and the Generalised Anxiety Disorder Assessment-73); and services will operate according to a 'stepped care model'.

'Low intensity practitioners' have some similarities in training to 'graduate mental health workers' whose role was proposed in The NHS Plan in 2000, although lessons have been learned from the problems associated with this previous initiative. ${ }^{4,5}$ These low intensity practitioners, who may have limited mental health experience, are trained to provide a wider range evidencebased brief interventions derived from cognitive behavioural therapy (CBT), such as facilitated self-help (computerised CBT and bibliotherapy), behavioural activation, and problem-solving, in addition to signposting to other community resources.

The more numerous 'high intensity practitioners' provide CBT to those who have not recovered with lower intensity treatments. Targets for supporting patients to return to work are being revisited in the new economic climate. Contrary to some reports, counselling and other treatment modalities can be integrated into or co-exist in parallel with the new IAPT teams. The ambitions of the IAPT programme to improve access to talking therapies to a wider population, including vulnerable groups, is bold and potentially of great importance to patients and their GPs; however, this poses some challenges too, not least that of horizontally integrating into primary care an essentially vertically organised programme of care focused on depression and anxiety. ${ }^{6}$

We suggest that the IAPT programme is best seen as a critical component of the wider project to improve primary care mental health. ${ }^{7}$ This vision needs to include services that break down the body-mind divide, locate specialist mental health workers in primary care settings, and enhance primarybased care for patients with complex conditions, such as psychosis, dementia, medically unexplained symptoms, personality disorder, and drug and alcohol problems - most of whom have significant comorbidity.

There will be roles for both new and established professionals, with primary care clinicians gaining advanced consultations skills and integrating CBT and other approaches into the consultation. There will also be a shift in language from biomedicalbased points of reference, to language that reflects both the challenge of engaging vulnerable groups and acceptance of the promotion of the values of recovery, selfcare, and social inclusion into mainstream general practice. The new psychological therapy services are vital for this wider project.

GPs, possibly through practice-based commissioning and 'Darzi' commissioning groups, can take advantage of the considerable flexibilities in design of IAPT services which can be applied at the local level. This engagement of primary care workers is particularly important, given the potential gaps in services and other operational problems that rapid introduction of a large new workforce in training may engender.

The gaps in care for complex cases, in coordination and in treatment modalities, are already emerging in first wave PCT sites (several per strategic health authority). The new IAPT services are designed primarily for people with depression and anxiety. Those people with comorbid personality disorder are seen as too difficult and complex, yet are not always accepted by some secondary mental health teams. People with severe depression not associated with psychosis or acute suicidal risk, or those with a severe degree of post-traumatic stress disorder or obsessive-compulsive disorder, and comorbid drug or alcohol problems can fall between teams.

There is little evidence on how to engage and treat people with comorbid long-term physical health problems, as they often resist mental health diagnoses. The new services are not specifically designed for young people (who, despite contact, fail to disclose mental health problems ${ }^{8}$ ) or for older adults who may face isolation and be homebound.

Even people with a relatively simple depressive episode will require coordination of different elements of care: medication management, talking therapy, and exercise. The new provision for psychological therapy is designed to complement antidepressants. Although prescribing rates continue to rise, and there are ongoing concerns that social causes are being medicalised, ${ }^{9}$ there is also still evidence of unmet need. ${ }^{10}$ Psychiatric advice about medication is not part of the basic IAPT model, and few services have so far established explicit links with psychiatrists.

Accommodation for the new therapists in general practice is another problem. Some practices have insufficient space and others choose to apply high service charges, resulting in reductions in budgets or relocation of therapists away from GP surgeries.

Probably the major gap is in the IAPT budget. The 2008-2011 programme, designed to reach $50 \%$ of primary care trusts, appears safe but the original hope of similar investment over 2011-2014 has disappeared. In regions that did not spread the investment across all PCTs, radical solutions involving redistribution are being considered. In some areas, provision of nonCBT modalities, such as counselling, has 
been reduced (or even cut completely) and there are no central plans to invest in a broader range of psychological therapies.

In addition to these gaps, there are also operational issues, which is not surprising given the extent and rapidity of investment. At practice level some previously productive relationships with independent counsellors and therapists have been disrupted. Relationships with new staff may take time to rebuild. Because the training for high intensity therapists involves 2 days per week, it will take 2 years to achieve full capacity; where expectations have been raised, waiting lists are common.

Initially, therapists in training need straightforward cases, yet experienced GPs need support with complex patients. Some referral pathways, dependent on telephone triage and assessments for suitability, are over complex and are being redesigned. Data collection requirements are immense. Although bespoke information systems have been designed, it has been challenging for practitioners to change practice to integrate this requirement seamlessly into therapeutic consultations.

For all these problems, the potential gain is great and most are solvable. We propose that a number of issues are given more priority: sharing and use of data, development of improved care pathways to try to achieve the goal of 'seamless care', and the development of liaison and consultancy with mental health services. Despite the formal and quite prescriptive service design requirements from the Department of Health, there is still considerable local flexibility. GPs need to be fully involved in the redesign of local services. In the long term, only local expertise will determine how successfully the new services function.

Integrated care with seemless pathways and more efficient shared care can be developed by:

- Ensuring that referral to the new IAPT services from GPs and others minimises the number of steps (and assessments) the patient has to negotiate before obtaining the right intensity of therapy. ${ }^{11}$ GPs should have freedom to refer directly to high intensity therapists if this is clearly what the patient requires. Additionally, the new 'single point of access' triage offered by some mental health services introduces an additional step and can deskill GPs.

- Commissioners ensuring that gaps and blocks between different services are addressed; for example, by linking together the sometimes fragmented elements of mental health care (drugs, alcohol, older people, child and adolescent services).

- New services implementing assertive but efficient systems of review that ensure that those not recovering with psychological therapy are reassessed and referred on if appropriate.

The data collected for individuals is highly specified, but its use is not. General practices require timely feedback from IAPT providers of clinical information about individuals which is transmitted automatically to electronic records. Analysis of data at practitioner and practice levels will allow practices to work with IAPT services to improve the flow of patients through the system and for GPs to target referral better.

Collaborative care and liaison will require the location of the new psychological therapists in general practice; opportunities for discussion of cases; rapid sharing of clinical data through entry of progress notes directly into practice electronic data systems; links with psychiatrists giving timely advice about psychotropic medication to GPs; and pragmatic, suitably trained, and experienced psychiatrists, community mental health nurses, and GPs with special interests providing advice and support to the new psychological therapists about complex patients with physical conditions, personality disorder, and substance misuse; thus creating 'primary care mental health teams'.

Perhaps most importantly, IAPT services supported by their GP leads will need to win over the hearts and minds of GPs, to increase GPs' knowledge of the wide range of options and to optimise referral patterns. GPs' current referral patterns for psychological reasons are influenced by patient requests and GPs' own self-rated abilities; however, they are also influenced by whether GPs believe the patient has capacity to receive therapy..$^{12}$ The latter may need to be addressed: the wide range of options means that there will be something for most people in the improving access to psychological therapies pot.

\section{Richard Byng,}

Senior Clinical Research Fellow, Primary Care Group, Peninsula Medical School, and GP Advisor for IAPT, South West Development Centre.

\section{Linda Gask,}

Professor of Primary Care Psychiatry, University of Manchester and Honorary Consultant Psychiatrist, Salford PCT.

\section{Provenance}

Commissioned; not peer reviewed

\section{Competing interests}

Richard Byng has a role in the south west region of England promoting links between general practice and the IAPT services. He has also been commissioned to carry out an evaluation of the services in the south west. Linda Gask is actively engaged in the implementation of IAPT in Salford.

\section{REFERENCES}

1. Improving Access to Psychological Therapies. About us. London: IAPT. http://www.iapt.nhs.uk/about (accessed 6 Aug 2009).

2. Kroenke K, Spitzer RL, Williams JB. The PHQ-9: validity of a brief depression severity measure. J Gen Intern Med 2001; 16(9): 606-613.

3. Spitzer RL, Kroenke K, Williams JB, Löwe B. A brief measure for assessing generalized anxiety disorder: the GAD-7. Arch Intern Med 2006; 166(10): 1092-1097.

4. Lester H, Freemantle N, Wilson S, et al. Cluster randomised controlled trial of the effectiveness of primary care mental health workers. Br J Gen Pract 2007; 57(536): 196-203.

5. Harkness E, Bower P, Gask L, Sibbald B. Retention and future job intentions of graduate primary care mental health workers: a newly developed role in England. J Health Serv Res Policy 2007; 12(Suppl 1): 18-22.

6. De Maeseneer J, Moosa S, Pongsupap Y, Kaufman A. Primary health care in a changing world. Br J Gen Pract 2008; 58(556): 806-809, i-ii.

7. New Ways of Working Primary Care Subgroup. New ways of working for primary care mental health: a briefing document. 2009. http://www.newwaysofworking.org.uk/ component/option,com_docman/task,cat_view/gid,213/Ite mid,412/ (accessed 17 Aug 2009).

8. Mauerhofer A, Berchtold A, Michaud P-A, Suris J-C. GPs' role in the detection of psychological problems of young people: a population-based study. Br J Gen Pract 2009; DOI: 10.3399/bjgp09X454115.

9. Macdonald S, Morrison J, Maxwell M, et al. 'A coal face option': GPs' perspectives on the rise in antidepressant prescribing. Br J Gen Pract 2009; DOI: 10.3399/bjgp09X454106

10. Cameron I, Reid I, Lawton K. Appropriateness of antidepressant prescribing: an observational study in a Scottish primary-care setting. Br J Gen Pract 2009: 59(566): 644-649.

11. Lovell K, Richards D. Multiple Access Points and Levels of Entry (MAPLE): ensuring choice, accessibility and equity for CBT services. Behav Cogn Psychother 2000; 28(4): 379-391.

12. Stavrou S, Cape J, Barker C. Decisions about referrals for psychological therapies: a matched-patient qualitative study.Br J Gen Pract 2009; DOI: 10.3399/bjgp09X454089.

DOI:10.3399/bjgp09X454043

\section{ADDRESS FOR CORRESPONDENCE}

\section{Richard Byng}

Peninsula Medical School, ITTC Building, Tamar Science Park, Davy Road, Plymouth, PL6 8BX, UK

Email: richard.byng@pms.ac.uk 